\title{
Rotational symmetry applied to boundary element computation for nuclear fusion plasma
}

\author{
M. Itagaki ${ }^{1}$, T. Ishimaru ${ }^{1} \& \mathrm{~K}$. Watanabe ${ }^{2}$ \\ ${ }^{I}$ Faculty of Engineering, Hokkaido University, Japan \\ ${ }^{2}$ National Institute for Fusion Science, Japan
}

\begin{abstract}
An efficient boundary element technique has been proposed to deal with a problem when geometry and boundary conditions have a rotational symmetry. In this technique, no boundary elements need to be defined on the symmetry surface. This idea has been applied to a problem of magnetic field in nuclear fusion plasma. A useful "linear transformation" for the rotational symmetry in the toroidal direction has been derived in terms of the $\mathrm{x}$-, $\mathrm{y}$ - and $\mathrm{z}$-components of vector potential in a 3-D Cartesian coordinate system. Applying this linear transformation to the discretized set of boundary integral equations, one can drastically reduce the size of matrix, the number of unknowns and then computing cost. Also, accurate solutions can be expected. Results of test calculations demonstrate the validity of the present formulation.

Keywords: boundary element method, rotational symmetry, vector potential, linear transformation, nuclear fusion plasma, Cauchy condition surface method, magnetic sensor.
\end{abstract}

\section{Introduction}

The present work is a part of the authors' research plan to develop an inverse analytic technique to identify the boundary shape of nuclear fusion plasma from signals of magnetic sensors located outside the plasma. For this purpose, the Cauchy condition surface (CCS) method [1] has already been established for a tokamak-type fusion device. The geometry of tokamak plasma is axisymmetric so that the analysis can be made in a 2-dimensional (2-D), $r-z$ system. On the other hand, 3-D analyses are required for non-axisymmetric plasma, e.g., in a helical type device such as the Large Helical Device (LHD). The application of 
the CCS method to a 3-D space analysis, however, becomes quite challenging because the 3-D system has a much larger number of unknowns than a 2-D system.

The authors have noticed that the LHD has a $1 / 5$ rotational symmetry. In Section 2 of the present paper, an efficient boundary element technique is given for solving a simple potential problem having rotational symmetry. When there is an $n$-fold rotational symmetry, the system matrix becomes a cyclic one. Because of this, the number of unknowns is reduced to $1 / n$. In this case, no boundary elements need to be defined on the symmetry surface.

In Section 3, this idea is extended to nuclear fusion plasma that has a rotational symmetry in the toroidal direction. A useful "linear transformation" is derived in terms of the $\mathrm{x}-, \mathrm{y}$ - and $\mathrm{z}$-components of vector potential in a Cartesian coordinate system. Applying this linear transformation to the discretized set of boundary integral equations, one can reduce the number of unknowns and then computing cost dramatically. Also, accurate solutions can be expected.

Numerical examples are shown in Section 4 to demonstrate the validity of the present formulation to deal with the rotational symmetry.

\section{Rotational symmetry in a simple potential problem}

First of all, one here considers a simple potential problem. Corresponding to the 2-D Laplace equation

$$
\frac{\partial^{2} u}{\partial x^{2}}+\frac{\partial^{2} u}{\partial y^{2}}=0
$$

the boundary integral equation is given by

$$
c_{i} u_{i}=\int_{\Gamma}\left(u_{i}^{*} \frac{\partial u}{\partial n}-u \frac{\partial u_{i}^{*}}{\partial n}\right) d \Gamma,
$$

which discretized form can be written in a matrix equation form [2]

$$
\mathbf{H u}=\mathbf{G q} \text {. }
$$

Here, the quantities $\mathbf{u}$ and $\mathbf{q}$ denote vectors of the Dirichlet condition $u$ and the Neumann condition $q=\partial u / \partial n$, respectively on the boundary $\Gamma$.

When the boundary $\Gamma$ is divided into $n$ segments, $\left(\Gamma_{1}, \Gamma_{2}, \cdots, \Gamma_{n}\right)$, eqn (3) is given by

$$
\left[\begin{array}{cccc}
\mathbf{H}_{1,1} & \mathbf{H}_{1,2} & \cdots & \mathbf{H}_{1, n} \\
\mathbf{H}_{2,1} & \mathbf{H}_{2,2} & \cdots & \mathbf{H}_{2, n} \\
\cdots & \cdots & \cdots & \cdots \\
\mathbf{H}_{n, 1} & \mathbf{H}_{n, 2} & \cdots & \mathbf{H}_{n, n}
\end{array}\right]\left\{\begin{array}{l}
\mathbf{u}_{1} \\
\mathbf{u}_{2} \\
\cdots \\
\mathbf{u}_{n}
\end{array}\right\}=\left[\begin{array}{cccc}
\mathbf{G}_{1,1} & \mathbf{G}_{1,2} & \cdots & \mathbf{G}_{1, n} \\
\mathbf{G}_{2,1} & \mathbf{G}_{2,2} & \cdots & \mathbf{G}_{2, n} \\
\cdots & \cdots & \cdots & \cdots \\
\mathbf{G}_{n, 1} & \mathbf{G}_{n, 2} & \cdots & \mathbf{G}_{n, n}
\end{array}\right]\left\{\begin{array}{c}
\mathbf{q}_{1} \\
\mathbf{q}_{2} \\
\cdots \\
\mathbf{q}_{n}
\end{array}\right\}
$$

using submatrices $\mathbf{H}_{i, j}$ and $\mathbf{G}_{i, j}$. If there is an $n$-fold rotational symmetry in the geometry as well as the boundary conditions under consideration, one finds 
$\mathbf{u}_{1}=\mathbf{u}_{2}=\cdots=\mathbf{u}_{n}$ and $\mathbf{q}_{1}=\mathbf{q}_{2}=\cdots=\mathbf{q}_{n}$. Also, the matrix on each side in eqn (4) becomes a cyclic one. That is, eqn (4) can be rewritten as

$$
\left[\begin{array}{cccc}
\mathbf{H}_{1,1} & \mathbf{H}_{1,2} & \cdots & \mathbf{H}_{1, n} \\
\mathbf{H}_{1, n} & \mathbf{H}_{1,1} & \cdots & \mathbf{H}_{1, n-1} \\
\cdots & \cdots & \cdots & \cdots \\
\mathbf{H}_{1,2} & \mathbf{H}_{1,3} & \cdots & \mathbf{H}_{1,1}
\end{array}\right]\left\{\begin{array}{l}
\mathbf{u}_{1} \\
\mathbf{u}_{1} \\
\cdots \\
\mathbf{u}_{1}
\end{array}\right\}=\left[\begin{array}{cccc}
\mathbf{G}_{1,1} & \mathbf{G}_{1,2} & \cdots & \mathbf{G}_{1, n} \\
\mathbf{G}_{1, n} & \mathbf{G}_{1,1} & \cdots & \mathbf{G}_{1, n-1} \\
\cdots & \cdots & \cdots & \cdots \\
\mathbf{G}_{1,2} & \mathbf{G}_{1,3} & \cdots & \mathbf{G}_{1,1}
\end{array}\right]\left\{\begin{array}{l}
\mathbf{q}_{1} \\
\mathbf{q}_{1} \\
\cdots \\
\mathbf{q}_{1}
\end{array}\right\} .
$$

Equation (5) can then be simplified as

$$
\left[\mathbf{H}_{1,1}+\mathbf{H}_{1,2}+\cdots+\mathbf{H}_{1, n}\right]\left\{\mathbf{u}_{1}\right\}=\left[\mathbf{G}_{1,1}+\mathbf{G}_{1,2}+\cdots+\mathbf{G}_{1, n}\right]\left\{\mathbf{q}_{1}\right\} .
$$

Now the matrix size and the number of unknowns are reduced to $1 / n^{2}$ and $1 / n$, respectively. It should be noted that in this case one need not to define any boundary elements on the symmetry surface, so that the periodic boundary conditions are naturally satisfied.

Suppose now $J$ mesh points, $\left(x_{j}^{(1)}, y_{j}^{(1)}\right),(j=1,2, \cdots, J)$, have been given for the boundary $\Gamma_{1}$ to generate the submatrices $\mathbf{H}_{1,1}$ and $\mathbf{G}_{1,1}$. Other mesh point coordinates

$$
\left(x_{j}^{(k)}, y_{j}^{(k)}\right), \quad(k=2,3, \cdots, n ; j=1,2, \cdots, J)
$$

to compute $\left[\mathbf{H}_{1,2}, \mathbf{H}_{1,3}, \cdots, \mathbf{H}_{1, n}\right]$ and $\left[\mathbf{G}_{1,2}, \mathbf{G}_{1,3}, \cdots, \mathbf{G}_{1, n}\right]$ are calculated using the well-known linear transformation

$$
\left\{\begin{array}{c}
x_{j}^{(k)}-x_{0} \\
y_{j}^{(k)}-y_{0}
\end{array}\right\}=\left[\begin{array}{cc}
\cos (k-1) \alpha & -\sin (k-1) \alpha \\
\sin (k-1) \alpha & \cos (k-1) \alpha
\end{array}\right]\left\{\begin{array}{c}
x_{j}^{(1)}-x_{0} \\
y_{j}^{(1)}-y_{0}
\end{array}\right\}
$$

with the angle $\alpha=2 \pi / n$ and the rotation axis $\left(x_{0}, y_{0}\right)$, as shown in fig. 1 .

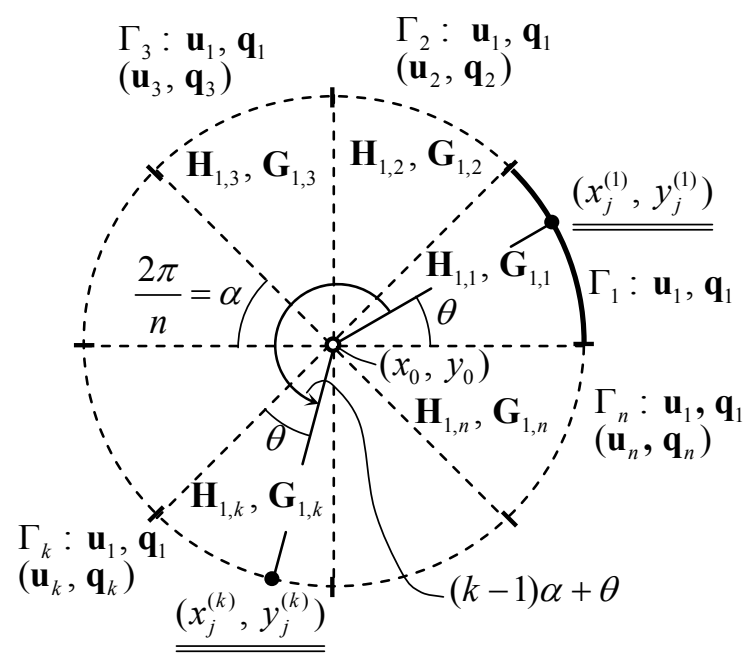

Figure 1: $\quad$ Boundary segmentation with $n$-fold rotational symmetry. 


\section{Rotational symmetry of 3-D vector potential}

The fundamental idea described in Section 2 is now applied to a problem of magnetic field in nuclear fusion plasma, which is described using vector potential in a 3-D space. This section describes how the rotational symmetry is introduced to reduce the number of unknowns in the 3-D CCS method formulation that is given by a set of boundary integral equations for points along the CCS and for the magnetic sensor positions. The boundary integral equations formulated in this work are all described in a Cartesian coordinate system. The reason for this is given in the Appendix. An outline of 3-D CCS method is found in the literature [3].

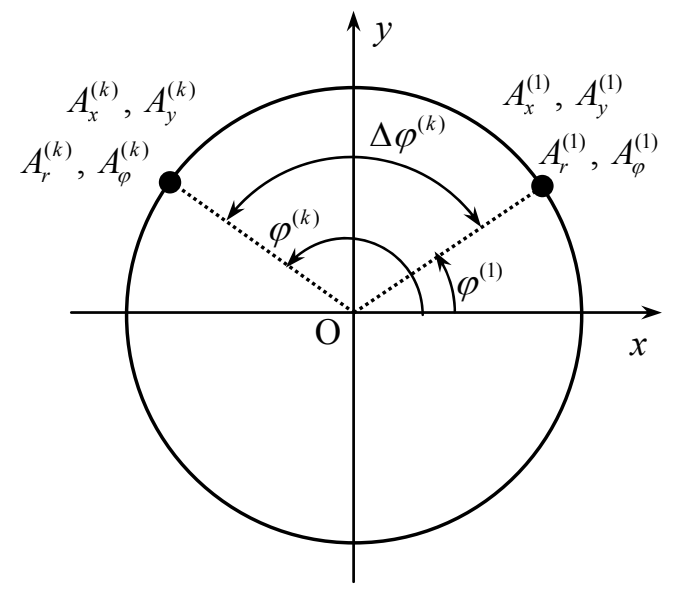

Figure 2: $\quad$ Rotational transformation of vector potentials.

\subsection{Linear transformation of vector potential}

Now one describes the vector potential $\left(A_{x}^{(k)}, A_{y}^{(k)}, A_{z}^{(k)}\right)$ in the $k$-th segment in terms of the vector potential $\left(A_{x}^{(1)}, A_{y}^{(1)}, A_{z}^{(1)}\right)$ in the first segment. The relationship between $\left(A_{x}^{(k)}, A_{y}^{(k)}\right)$ and $\left(A_{r}^{(k)}, A_{\varphi}^{(k)}\right)$ in the different coordinate systems are described using the toroidal angle $\varphi^{(k)}$, as

$$
\left(\begin{array}{c}
A_{x}^{(k)} \\
A_{y}^{(k)}
\end{array}\right)=\left(\begin{array}{cc}
\cos \varphi^{(k)} & -\sin \varphi^{(k)} \\
\sin \varphi^{(k)} & \cos \varphi^{(k)}
\end{array}\right)\left(\begin{array}{c}
A_{r}^{(k)} \\
A_{\varphi}^{(k)}
\end{array}\right) .
$$

Considering the rotational symmetry, i.e., $A_{r}^{(k)}=A_{r}^{(1)}$ and $A_{\varphi}^{(k)}=A_{\varphi}^{(1)}$, one finds

$$
\left(\begin{array}{c}
A_{x}^{(k)} \\
A_{y}^{(k)}
\end{array}\right)=\left(\begin{array}{cc}
\cos \varphi^{(k)} & -\sin \varphi^{(k)} \\
\sin \varphi^{(k)} & \cos \varphi^{(k)}
\end{array}\right)\left(\begin{array}{c}
A_{r}^{(1)} \\
A_{\varphi}^{(1)}
\end{array}\right) .
$$


With the relationship between $\left(A_{r}^{(1)}, A_{\varphi}^{(1)}\right)$ and $\left(A_{x}^{(1)}, A_{y}^{(1)}\right)$, eqn (9) becomes

$$
\left(\begin{array}{c}
A_{x}^{(k)} \\
A_{y}^{(k)}
\end{array}\right)=\left(\begin{array}{cc}
\cos \varphi^{(k)} & -\sin \varphi^{(k)} \\
\sin \varphi^{(k)} & \cos \varphi^{(k)}
\end{array}\right)\left(\begin{array}{cc}
\cos \varphi^{(1)} & \sin \varphi^{(1)} \\
-\sin \varphi^{(1)} & \cos \varphi^{(1)}
\end{array}\right)\left(\begin{array}{l}
A_{x}^{(1)} \\
A_{y}^{(1)}
\end{array}\right) .
$$

This formula can be transformed into

$$
\left(\begin{array}{c}
A_{x}^{(k)} \\
A_{y}^{(k)}
\end{array}\right)=\left(\begin{array}{cc}
\cos \Delta \varphi^{(k)} & -\sin \Delta \varphi^{(k)} \\
\sin \Delta \varphi^{(k)} & \cos \Delta \varphi^{(k)}
\end{array}\right)\left(\begin{array}{c}
A_{x}^{(1)} \\
A_{y}^{(1)}
\end{array}\right)
$$

using the "additional theorem" of trigonometric functions with a rotation angle

$$
\Delta \varphi^{(k)}=\varphi^{(k)}-\varphi^{(1)} \text {. }
$$

Including the $z$-component, one finally obtains

$$
\left(\begin{array}{c}
A_{x}^{(k)} \\
A_{y}^{(k)} \\
A_{z}^{(k)}
\end{array}\right)=\left(\begin{array}{ccc}
\cos \Delta \varphi^{(k)} & -\sin \Delta \varphi^{(k)} & 0 \\
\sin \Delta \varphi^{(k)} & \cos \Delta \varphi^{(k)} & 0 \\
0 & 0 & 1
\end{array}\right)\left(\begin{array}{c}
A_{x}^{(1)} \\
A_{y}^{(1)} \\
A_{z}^{(1)}
\end{array}\right) .
$$

This is the "linear transformation" from $\left(A_{x}^{(1)}, A_{y}^{(1)}, A_{z}^{(1)}\right)$ to $\left(A_{x}^{(k)}, A_{y}^{(k)}, A_{z}^{(k)}\right)$ in a Cartesian coordinate system.

\subsection{Application to boundary element equations in 3-D CCS method}

The set of boundary integral equations for the 3-D CCS method can also be transformed into a matrix equation

$$
\mathbf{H u}=\mathbf{G q},
$$

where

$$
\mathbf{u}=\left\{\mathbf{A}_{x}^{(1)}, \mathbf{A}_{y}^{(1)}, \mathbf{A}_{z}^{(1)}, \cdots, \mathbf{A}_{x}^{(n)}, \mathbf{A}_{y}^{(n)}, \mathbf{A}_{z}^{(n)}\right\}^{T}
$$

and

$$
\mathbf{q}=\left\{\frac{\partial \mathbf{A}_{x}^{(1)}}{\partial n}, \frac{\partial \mathbf{A}_{y}^{(1)}}{\partial n}, \frac{\partial \mathbf{A}_{z}^{(1)}}{\partial n}, \cdots, \frac{\partial \mathbf{A}_{x}^{(n)}}{\partial n}, \frac{\partial \mathbf{A}_{y}^{(n)}}{\partial n}, \frac{\partial \mathbf{A}_{z}^{(n)}}{\partial n}\right\}^{T} .
$$

Here, $\left(\mathbf{A}_{x}^{(k)}, \mathbf{A}_{y}^{(k)}, \mathbf{A}_{z}^{(k)}\right)$ means the set of all vector potentials within the $k$-th segment. The LHS of eqn (13), for example, can be written as

Hu

$$
=\left[\begin{array}{ccccccc}
\mathbf{H}_{11} & \mathbf{H}_{12} & \mathbf{H}_{13} & \cdots & \mathbf{H}_{1,3 n-2} & \mathbf{H}_{1,3 n-1} & \mathbf{H}_{1,3 n} \\
\mathbf{H}_{21} & \mathbf{H}_{22} & \mathbf{H}_{23} & \cdots & \mathbf{H}_{2,3 n-2} & \mathbf{H}_{2,3 n-1} & \mathbf{H}_{2,3 n} \\
\mathbf{H}_{31} & \mathbf{H}_{32} & \mathbf{H}_{33} & \cdots & \mathbf{H}_{3,3 n-2} & \mathbf{H}_{3,3 n-1} & \mathbf{H}_{3,3 n} \\
\vdots & \vdots & \vdots & & \vdots & \vdots & \vdots \\
\mathbf{H}_{l, 1} & \mathbf{H}_{l, 2} & \mathbf{H}_{l, 3} & & \mathbf{H}_{l, 3 n-2} & \mathbf{H}_{l, 3 n-1} & \mathbf{H}_{l, 3 n} \\
\vdots & \vdots & \vdots & & \vdots & \vdots & \vdots \\
\mathbf{H}_{L-1,1} & \mathbf{H}_{L-1,2} & \mathbf{H}_{L-1,3} & \cdots & \mathbf{H}_{L-1,3 n-2} & \mathbf{H}_{L-1,3 n-1} & \mathbf{H}_{L-1,3 n} \\
\mathbf{H}_{L, 1} & \mathbf{H}_{L, 2} & \mathbf{H}_{L, 3} & \cdots & \mathbf{H}_{L, 3 n-2} & \mathbf{H}_{L, 3 n-1} & \mathbf{H}_{L, 3 n}
\end{array}\right]\left(\begin{array}{c}
\mathbf{A}_{x}^{(1)} \\
\mathbf{A}_{y}^{(1)} \\
\mathbf{A}_{z}^{(1)} \\
\vdots \\
\mathbf{A}_{x}^{(n)} \\
\mathbf{A}_{y}^{(n)} \\
\mathbf{A}_{z}^{(n)}
\end{array}\right),
$$


where $L$ denotes the number of lines that depends on the number of nodal points on the CCS and the number of magnetic sensors as well. Assuming an $n$-fold rotational symmetry, i.e., applying eqn (12) to the vector on the RHS in eqn (16), eqn (16) is described only using $\mathbf{A}_{x}^{(1)}, \mathbf{A}_{y}^{(1)}$ and $\mathbf{A}_{z}^{(1)}$. For instance, the $l$ th line of Hu can be written in the form

$$
\begin{aligned}
& (\mathbf{H u})_{l} \\
& =\left[\begin{array}{l}
\sum_{k=1}^{n}\left(\mathbf{H}_{l, 3 k-2} \cos \Delta \varphi^{(k)}+\mathbf{H}_{l, 3 k-1} \sin \Delta \varphi^{(k)}\right), \\
\sum_{k=1}^{n}\left(-\mathbf{H}_{l, 3 k-2} \sin \Delta \varphi^{(k)}+\mathbf{H}_{l, 3 k-1} \cos \Delta \varphi^{(k)}\right), \\
\sum_{k=1}^{n} \mathbf{H}_{l, 3 k}
\end{array}\right]\left(\begin{array}{c}
\mathbf{A}_{x}^{(1)} \\
\mathbf{A}_{y}^{(1)} \\
\mathbf{A}_{z}^{(1)}
\end{array}\right) .
\end{aligned}
$$

The Gq on the RHS of eqn (13) can also be rewritten in the same way. Both the numbers of columns and unknowns can be reduced to $1 / n$ if only giving the rotational angle $\Delta \varphi^{(k)}$.

When a singular point $i$ is located on the CCS, the boundary integral equation for each of $\mathbf{A}_{x}, \mathbf{A}_{y}$ and $\mathbf{A}_{z}$ holds independently, and all equations commonly use the same fundamental solution. Because of this, in eqn (16) the portion related to the $\mathrm{CCS}$ represents a $3 n \times 3 n$ square matrix equation:

$$
\begin{aligned}
& \mathbf{H u}^{C C S} \\
& =\left[\begin{array}{ccccccc}
\mathbf{H}_{11} & 0 & 0 & \cdots & \mathbf{H}_{1,3 n-2} & 0 & 0 \\
0 & \mathbf{H}_{22} & 0 & \cdots & 0 & \mathbf{H}_{2,3 n-1} & 0 \\
0 & 0 & \mathbf{H}_{33} & \cdots & 0 & 0 & \mathbf{H}_{3,3 n} \\
\vdots & \vdots & \vdots & & \vdots & \vdots & \vdots \\
\mathbf{H}_{3 n-2,1} & 0 & 0 & \cdots & \mathbf{H}_{3 n-2,3 n-2} & 0 & 0 \\
0 & \mathbf{H}_{3 n-1,2} & 0 & \cdots & 0 & \mathbf{H}_{3 n-1,3 n-1} & 0 \\
0 & 0 & \mathbf{H}_{3 n, 3} & \cdots & 0 & 0 & \mathbf{H}_{3 n, 3 n}
\end{array}\right]\left(\begin{array}{c}
\mathbf{A}_{x}^{(1)} \\
\mathbf{A}_{y}^{(1)} \\
\mathbf{A}_{z}^{(1)} \\
\vdots \\
\mathbf{A}_{x}^{(n)} \\
\mathbf{A}_{y}^{(n)} \\
\mathbf{A}_{z}^{(n)}
\end{array}\right) .
\end{aligned}
$$

Then, introducing the rotational symmetry, eqn (18) can be simplified as

$$
\begin{aligned}
& \mathbf{H u}_{123}^{C C S} \\
& =\left[\begin{array}{ccc}
\sum_{k=1}^{n}\left(\mathbf{H}_{1,3 k-2} \cos \Delta \varphi^{(k)}\right) & \sum_{k=1}^{n}\left(-\mathbf{H}_{1,3 k-2} \sin \Delta \varphi^{(k)}\right) & 0 \\
\sum_{k=1}^{n}\left(\mathbf{H}_{2,3 k-1} \sin \Delta \varphi^{(k)}\right) & \sum_{k=1}^{n}\left(\mathbf{H}_{2,3 k-1} \cos \Delta \varphi^{(k)}\right) & 0 \\
0 & 0 & \sum_{k=1}^{n} \mathbf{H}_{3,3 k}
\end{array}\right]
\end{aligned}
$$


Finally, the equations for points on the CCS as well as the ones for the magnetic sensor positions are solved simultaneously. Once all the values on the CCS are known, one can calculate the magnetic field for arbitrary points.

\section{Numerical example}

One here considers a problem to model the non-axisymmetric plasma in the LHD, a helical-type device. The three components of magnetic field in the LHD were reconstructed using the 3-D CCS method. As the magnetic field profile is not axisymmetric, this problem is challenging and requires a large number of unknowns.

The results were compared with the reference solution obtained using the HINT code [4]. Figure 3 shows an example of the reference solution obtained using the HINT code. This figure gives the contours of the $r$-component of the magnetic field $B_{r}$ on the $r-z$ plane at the toroidal angle of $18 \mathrm{deg}$.

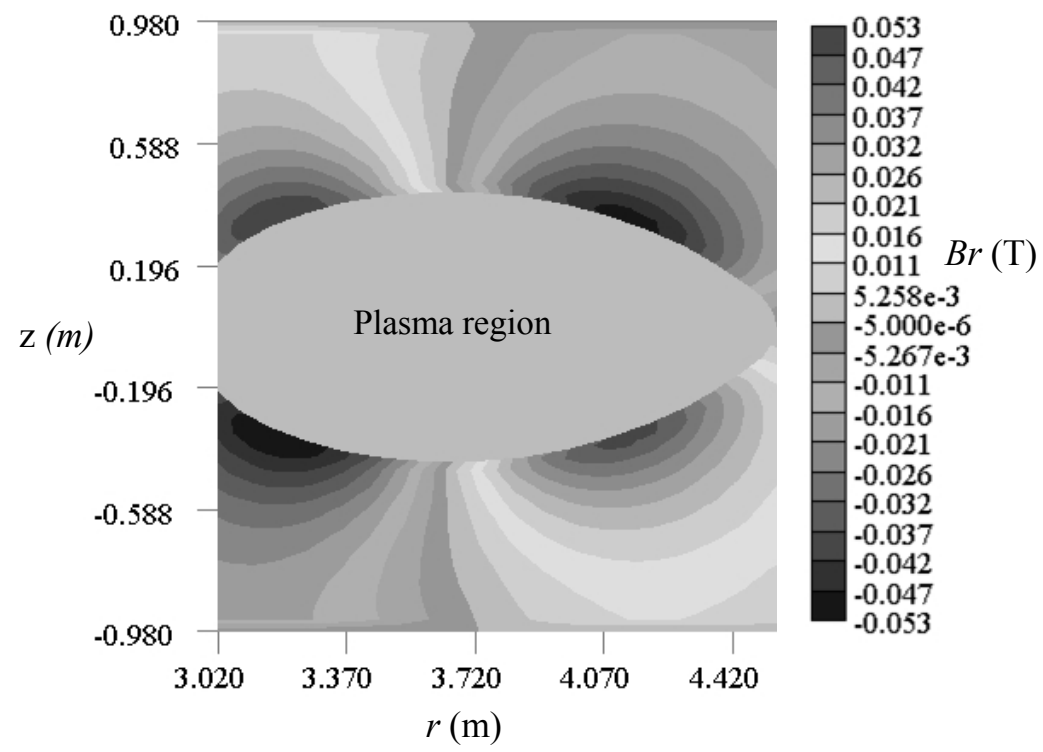

Figure 3: Reference profile of magnetic field $B_{r}$ at $\varphi=18 \mathrm{deg}$.

For inverse analyses, one here assumes that 20 magnetic flux loop sensors and 451 magnetic field sensors are arranged outside the plasma. Each of field sensors is hypothetically assumed to detect all of the three components of magnetic field. In this case the number of sensor signals is $451 \times 3+20=1373$. A tube-shaped CCS was placed within a domain that can be supposed to be inside the actual plasma. 
First, a CCS that covers 360-deg. was divided into 160 boundary elements (Case A). As the number of unknowns, 8640, is larger than the number of equations, 5693, as shown in Table 1, it was impossible to solve this problem.

Next, considering a 1/5 rotational symmetry, only 72-deg. portion of the CCS tube was modelled and this portion was divided into 32 boundary elements (Case B). Figure 4 shows the reconstructed contours of the $r$-component of the magnetic field $B_{r}$ on the $r-z$ plane at the toroidal angle of $18 \mathrm{deg}$., which correspond to the reference contours in fig. 3. As the magnetic fields computed using the CCS method has no physical meaning inside the plasma boundary [3], they are not drawn inside the plasma in fig. 4. This reconstructed field profile agrees well with the reference one in fig. 3.

Table 1: $\quad$ Calculation condition for plasma in LHD.

\begin{tabular}{|c|c|c|c|}
\hline Case & A & B & C \\
\hline Rotational symmetry & $360-$ deg. & $1 / 5$ symmetry & $360-$ deg. \\
\hline No. of sensor signals & 1373 & 1373 & 1373 \\
\hline No. of boundary elements & 160 & 32 & 40 \\
\hline No. of equations & 5693 & 2237 & 2453 \\
\hline No. of unknowns & 8640 & 1728 & 2160 \\
\hline Solution accuracy & Not solved & Acceptable & Poor \\
\hline
\end{tabular}
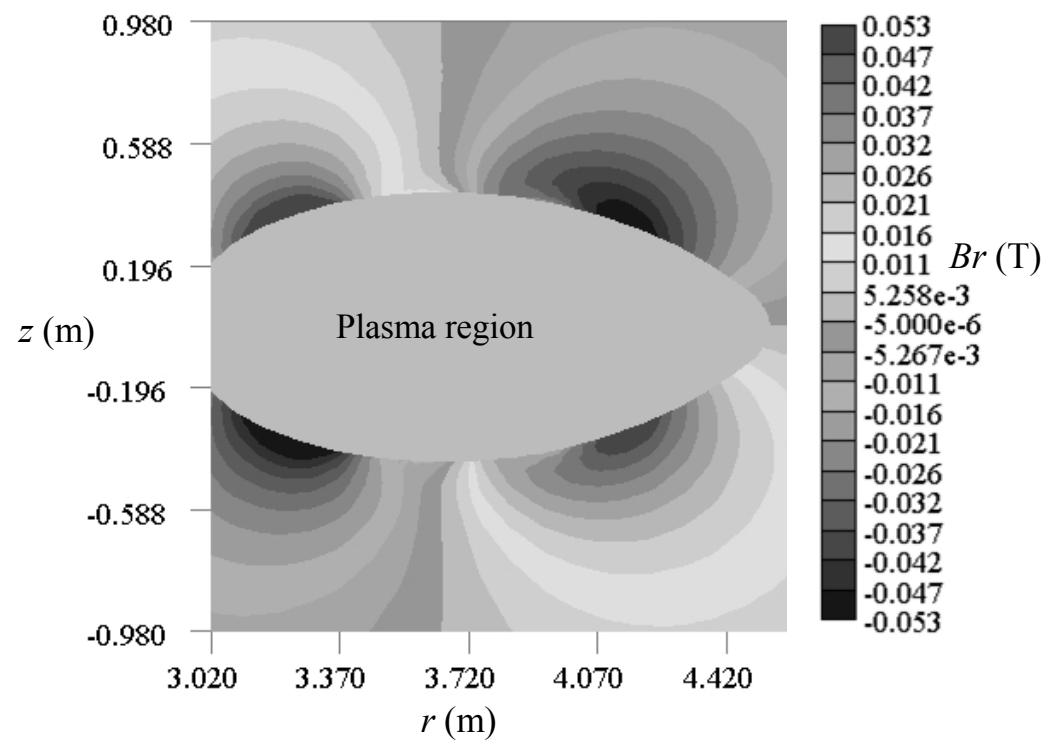

Figure 4: Profile of reconstructed magnetic field $B_{r}$ at $\varphi=18 \mathrm{deg}$. (Case B). 
When one took only 40 boundary elements on the CCS for 360-deg. (Case C), the number of unknowns is of course smaller than that of equations. Unfortunately, however the accuracy of the reconstructed solution in this case was very poor, as shown in fig. 5. That is, without considering the rotational symmetry, it is difficult to realize a good accuracy in the 3-D analysis of nonaxisymmetric plasma, which requires a large number of unknowns.

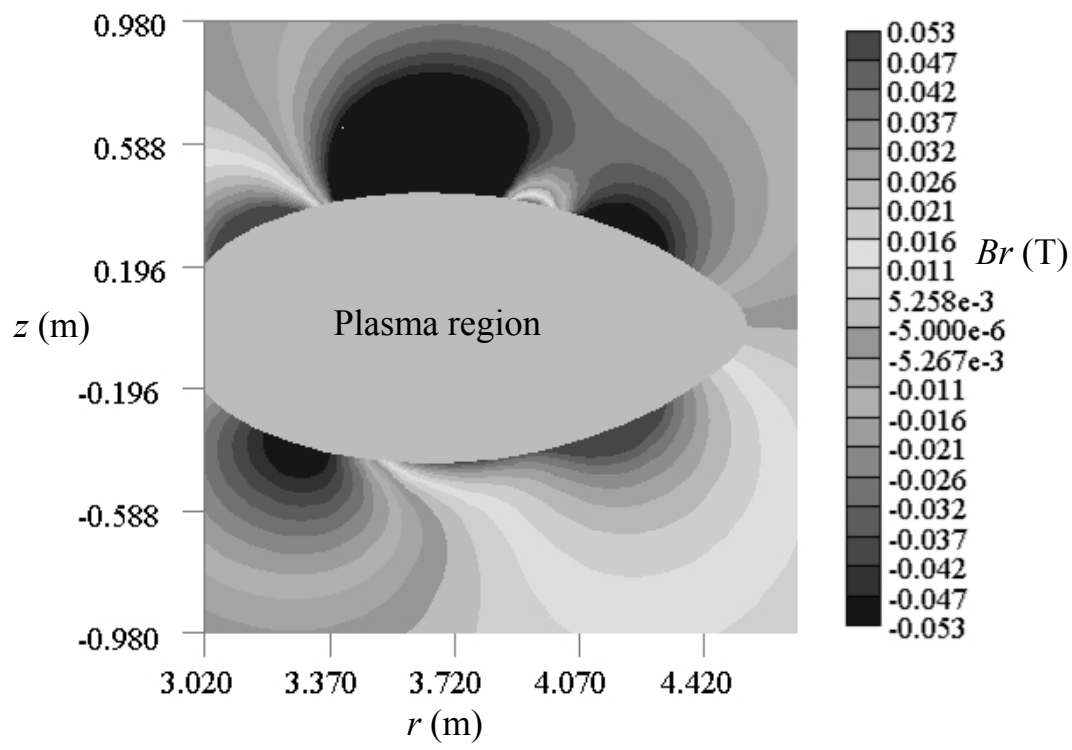

Figure 5: Profile of reconstructed magnetic field $B_{r}$ at $\varphi=18 \mathrm{deg}$. (Case C).

\section{Conclusion}

An efficient boundary element technique has been applied to problems of magnetic field in 3-D nuclear fusion plasma that has a rotational symmetry in the toroidal direction. In this technique, no boundary elements need to be defined on the symmetry surface. The linear transformation in terms of the $\mathrm{x}-, \mathrm{y}-$ and $\mathrm{z}-$ components of 3-D vector potential plays an important role to reduce the matrix size and the number of unknowns in the discretized set of boundary integral equations. This enables one to realize not only the reduction of the computing cost but the improvement of numerical solution accuracy.

Results of test calculations for nuclear fusion plasmas in a helical-type device demonstrate the validity of the present formulation. It has been found that one should incorporate the rotational symmetry into the analysis of nonaxisymmetric 3-D plasma in order to obtain an acceptable accuracy of the solution. 


\section{Acknowledgements}

This research was supported by the Ministry of Education, Culture, Sports, Science and Technology, Grant-in-Aid for Scientific Research (C), 20560762, 2009. This work was also performed with the support and under the auspices of the NIFS Collaboration Research Program (NIFS08KLHH308).

\section{Appendix: Why the boundary integral equations in this work are described in a Cartesian coordinate system?}

The vector Laplacian in a Cartesian coordinate system has a simple relationship

$$
\left(\nabla^{2} \boldsymbol{A}\right)_{k}=\nabla^{2} A_{k} \quad(k=x, y, z) .
$$

That is, the vector Laplacian can be given by a set of the scalar Laplacian of each Cartesian scalar component. In a cylindrical or a spherical coordinate system, on the other hand, the expression of the vector Laplacian is not so straightforward. In a cylindrical system, for example, the components of the vector Laplacian are written in complicated forms, as

$$
\begin{aligned}
& \left(\nabla^{2} \boldsymbol{A}\right)_{r}=\nabla^{2} A_{r}-\frac{2}{r^{2}} \frac{\partial A_{\varphi}}{\partial \varphi}-\frac{A_{r}}{r^{2}}, \\
& \left(\nabla^{2} \boldsymbol{A}\right)_{\varphi}=\nabla^{2} A_{\varphi}+\frac{2}{r^{2}} \frac{\partial A_{r}}{\partial \varphi}-\frac{A_{\varphi}}{r^{2}}
\end{aligned}
$$

and

$$
\left(\nabla^{2} A\right)_{z}=\nabla^{2} A_{z}
$$

If one uses this coordinate system, the boundary integral equations corresponding to $r$ - and $\varphi$-components will include domain integral terms. To realize a boundary-only integral formulation, it is better not to use a cylindrical or a spherical system. Because of this, the authors adopt a 3-D Cartesian coordinate system for the analysis to obtain the 3-D distribution of vector potential. However, it is easy to transform the result, once calculated in a Cartesian coordinate system, into one in another coordinate system.

\section{References}

[1] Kurihara, K, Fusion Eng. Des., 51-52, pp.1049-1057, 2000.

[2] Brebbia, C.A., "The Boundary Element Method for Engineers", Pentech Press, London, 1978

[3] Itagaki, M., Maeda, T., Wakasa, A., Watanabe, K., Proc. BEM/MRM XXXI, Southampton, pp.397-404, 2009.

[4] Harafuji, K., Hayashi T., Sato T., J. Comput. Phys., 81, pp.169-192, 1989. 